\title{
Pre- and post-infection activity of new fungicides against Botrytis cinerea and other fungi causing decay of table grapes
}

\author{
Ricardo A. Serey, René Torres, and Bernardo A. Latorre ${ }^{1}$ \\ Facultad de Agronomía e Ingeniería Forestal, Pontificia Universidad Católica de Chile \\ Casilla 306-22, Santiago, Chile
}

\begin{abstract}
R.A. Serey, R. Torres, and B.A. Latorre. 2007. Pre- and post-infection activity of new fungicides against Botrytis cinerea and other fungi causing decay of table grapes. Cien. Inv. Agr. 34(3):215-224. Pre- and post-harvest diseases restrict table grape production and exports (Vitis vinifera $\mathrm{L}$.) in Chile, with the most important disease being grey mold (Botrytis cinerea). In addition, rot due to Aspergillus niger, Cladosporium herbarum, Penicillium expansum, and Rhizopus stolonifer frequently occurs. The pre- and post-infection activity of fungicides against these pathogens was studied on Thompson Seedless table grapes. Detached, mature, berries were used, and inoculations were performed with $20 \mu \mathrm{L}$ of a $10^{6}$ spores $\cdot \mathrm{mL}^{-1}$ suspension placed on three punctures aseptically made at the calyx end of each berry. Fungicides used (per liter) were boscalid $(600 \mathrm{mg})$, boscalid $(200 \mathrm{mg})+$ pyraclostrobin $(100 \mathrm{mg})$, boscalid $(200 \mathrm{mg})+$ kresoxim methyl $(100 \mathrm{mg})$, cyprodinil $(60 \mathrm{mg})+$ fludioxonil $(40 \mathrm{mg})$, BAS $600 \mathrm{KBF}(100$ $\mathrm{mg})+$ metrafenone $(150 \mathrm{mg})$, BAS $600 \mathrm{KBF}(200 \mathrm{mg})+$ boscalid $(300 \mathrm{mg})$, BAS $600 \mathrm{KBF}$ $(100 \mathrm{mg})+$ pyraclostrobin $(100 \mathrm{mg})$, and captan $(400 \mathrm{mg})$. Each fungicide was applied either by drop $\left(12 \mu \mathrm{L} \cdot\right.$ berry $\left.^{-1}\right)$ placed on three punctures made with a sterile hypodermic needle or by $60 \mathrm{~s}$ immersion. Berries were then incubated in humid chambers at $20^{\circ} \mathrm{C}$. The pre-infection (protection) activity of the fungicides varied considerably among the pathogens tested and was found to be significant $(\mathrm{p}<0.001)$ and, with one exception $($ A. niger $)$, it was significantly $(\mathrm{p}<$ 0.002 ) affected by the application method. The interaction between fungicide and application method was only significant $(\mathrm{p}<0.001)$ for $R$. stolonifer at $48 \mathrm{~h}$ post treatment. In general, pre-infection activity gave 0 to 4 days protection after drop applications and 0 to 21 days after immersion treatments. The post-infection (curative) activity varied among pathogens and fungicide treatments. However, it was always below $24 \mathrm{~h}$.
\end{abstract}

Key words: Blue mold, curative activity, gray mold, protection activity, sour rot, Vitis vinifera.

\section{Introduction}

Decays are one of the main factors restricting the production and commercialization of table grapes (Vitis vinifera L.) in Chile and other countries (Franck et al., 2005; Lydakis and Aked; 2003; Lichter et al., 2002). Important economic losses usually occur during harvest, cold storage, and transportation of Chilean table grapes to markets in North America, Europe and Asia (Franck et al., 2005; Donoso and Latorre, 2006).

Received 07 April 2007. Accepted 11 August 2007.

'Corresponding author: blatorre@uc.cl
Botrytis cinerea Pers. is the most important pathogen affecting table grape production in Chile. Infections start from the inoculum present in the vineyard which can develop into latent infections with disease appearing later in packed table grapes during storage and transportation. Additionally, pre and post harvest decay caused by Aspergillus niger Tiegh, Cladosporium herbarum (Pers.) Link, Penicillium expansum Link and Rhizopus stolonifer (Ehrenb.) Vuill, has been reported in Chile and elsewhere (Hewitt, 1988; Zahavi et al., 2000; Latorre et al., 2002b). 
Fungicide treatments applied in the vineyard are important to prevent decay development at harvest or during post-harvest (Latorre et al., 2001; Franck et al., 2005). However, registration restrictions, tolerances established by import countries, and the development of resistant strains limit their use in table grapes and other fruit crops (Latorre et al., 1994; Latorre et al., 2002a; Errampalli and Crnko, 2004; Sallato and Latorre, 2006). Therefore, new, effective fungicide treatments with the lowest possible toxicological risk are required, as has been proposed elsewhere (Adaskaveg et al., 2005; Förster et al., 2007; McGrath, 2004).

Fungicides can provide disease control through both pre- and post-infection activity. Pre-infection activity is commonly known as protectant (preventive) activity and postinfection activity comprises a curative action that can involve both pre- and post-symptom expression activities.

The modes of actions of the fungicides used in this study are varied. Anilinopyrimidines inhibit methionine biosynthesis and secretion of hydrolytic enzymes. Carboximides inhibit succinate dehydrogenase in the cell respiration process. Phenylpyrrol inhibits histidine quinase. Phthalimides are multisite inhibitors. Strobilurines (Qo inhibitor fungicides, QoI) act at the quinone binding site of the cytochrome bc1 complex in the mitochondrial cell membrane. Triazolopyrimidins have an unknown mode of action (McGrath, 2004; FRAC, 2007).

Understanding a fungicide's mode of action and also whether it has activity both pre- and post-infection contributes considerably to improved control efficiency through optimizing application timing based on the host-pathogeninteractions in table grapes and other hosts (Szkolnik, 1978; Jones and Latorre, 1985; Wong and Wilcox, 2001; Rebollar-Alviter et al., 2007).

Therefore, the objectives of this study were to evaluate, under laboratory conditions, the preinfection and post-infection activity of boscalid, captan, cyprodinil, and the new fungicide BAS600 against B. cinerea, P. expansum, $R$. stolonifer, A. niger and $C$. herbarum, which are the main filamentous fungi associated with pre- and post-harvest decay of table grapes in Chile.

\section{Materials and methods}

\section{Table grapes}

All the experiments were conducted on healthy and mature 'Thompson Seedless' table grapes (total soluble solids $>16 \%$ ). Berries with their pedicel intact and without fungicide treatments were obtained from a commercial farm. Prior to every experiment, the berries were superficially disinfected with $0.025 \%$ sodium hypochlorite for $3 \mathrm{~min}$. They were washed in tap water and were aseptically distributed on grids in polyethylene chambers $(34 \mathrm{x} 25 \mathrm{x}$ $13 \mathrm{~cm})$ at $20^{\circ} \mathrm{C}$ and $93-96 \%$ relative humidity (RH). The RH was obtained by moistening a double paper layer placed at the bottom of each chamber. Relative humidity was verified with a HOBO sensor (Bourne, Massachussets, USA), located inside the chamber.

\section{Isolation and inoculation}

Isolates of B. cinerea, $P$. expansum, and $A$. niger were obtained from 'Thompson Seedless' table grapes. Cladosporium herbarum was obtained from 'Red Globe' berries, and $R$. stolonifer from strawberries. Pure cultures were obtained by sub-cultivating hyphal tips in potato dextrose agar acidified with $0.5 \mathrm{~mL} \cdot \mathrm{L}^{-1}$ of $90 \%$ lactic acid (APDA) at $20^{\circ} \mathrm{C}$. All isolates were pathogenic to table grapes.

The inoculum was prepared with spores obtained from 7 to 15 day old cultures in APDA. To avoid spore clusters that may impede a uniform distribution of the inoculum, the spores were suspended in $0.05 \%$ of Tween 80 in sterile distilled water. The final concentration was adjusted to $10^{6}$ spores $\cdot \mathrm{mL}^{-1}$ with the aid of a hemacytometer. An aliquot $(20 \mu \mathrm{L})$ from the respective spore suspension was delivered on the surface of each berry after they were aseptically wounded with a hypodermic needle (at 2-3 mm depth) at the calyx end. Wounded berries were used for drop and immersion fungicide treatments. These inoculation methods were done to mimic damage that berries may 
naturally suffer in the field and should allow the spore to be attached to the surface of the berry, germinate, develop a germ tube and possible an appressorium before penetration.

\section{Fungicides}

The fungicides used were: 1. Boscalid (Cantus $50 \%$ WG, BASF, Germany), 2. Boscalid combined with pyraclostrobin (Bellis WG; $25.2+12.8 \%$, respectively, BASF, Germany), 3. Boscalid combined with kresoxim methyl (BAS 517 SC, $10.0+20.0 \%$, respectively, BASF, Germany), 4. Cyprodinil combined with fludioxonil (Swicth WG, 37.5 + 25.0\% respectively, Syngenta Crop Protection, Switzerland), 5. BAS $600 \mathrm{KBF}$ at $20 \%$ in combination with metrafenone (BAS 560 $50 \%$ SC, BASF, Germany), 6. BAS $600 \mathrm{KBF}$ at $20 \%$ in combination with boscalid (Cantus $50 \%$ WG, BASF, Germany), 7. BAS $600 \mathrm{KBF}$ al $20 \%$ combined with pyraclostrobin (Comet 25\% EC, BASF, Germany), and 8. Captan (Captan 80 WP, BASF Chile S.A.). The rates used represent current rates for pre-harvest use against $B$. cinerea on table grapes (Table 1). Pre-infection activity

The pre-infection (protectant) activity was the maximum period of time post-treatment where fungicides were able to protect berries from infection with mean control efficacy $\geq 75 \%$. For this purpose, two tests were performed: 1 . Berries were inoculated 0, 12, 24, 48 and $96 \mathrm{~h}$ after depositing a drop of $12 \mu \mathrm{L}$ of the respective fungicide suspension over three wounds made at the calyx end of each berry. 2. Berries were wounded and inoculated 1, 2, 7, 14 and 21 days after treating berries by immersion for $60 \mathrm{~s}$ in the fungicide suspension. The diameter of the lesion developed was determined 2 to 3 days after inoculation.

\section{Post-infection activity}

The post-infection activity (eradication activity) was defined as the maximum post-inoculation period for applying a fungicide treatment and

Table 1. Fungicides and fungicide mixtures used in this study.

Cuadro 1. Fungicidas y mezclas fungicidas empleadas en este estudio.

\begin{tabular}{lllc}
\hline & Fungicides & & Concentration \\
$\mathrm{mg} \cdot \mathrm{L}^{-1}$
\end{tabular}

${ }^{1} \mathrm{SC}$, concentrate suspension; WG, granulate dispersible; EC, emulsifiable concentrate; WP, wettable powder.

${ }^{2}$ The mode of action in the biosynthetic processes of fungi are: Anilinopyrimidines inhibit methionine biosynthesis and secretion of hydrolytic enzymes. Carbaximides inhibit succinate dehydrogenase in the cell respiration process. Phenylpyrrol inhibits histidine quinase. Phthalimides are multisite inhibitors. Strobilurines (Qo inhibitor fungicides, QoI) act at the quinone binding site of the cytochrome bc1 complex in the mitochondrial cell membrane. Triazolopyrimidins have an unknown mode of action (FRAC, 2007).

${ }^{3}$ Rates were expressed as active ingredients (a.i.) and were suspended in water before use.

${ }^{\prime} S C$, suspensión concentrada; WG, gránulos dispersables; $E C$, concentrado emulsionable; $W P$, polvo mojable.

${ }^{2}$ Los modos de acción bioquímicos son: Anilinopirimdinas inhiben la biosíntesis de metionina y la secreción de enzimas hidroíticas. Caboxamidas inhiben la enzima sucinato dehidrogenasa en el proceso de respiración celular. Fenilpirrol (Phenylpyrrol) inhiben la enzima histidina quinasa. Phthalamidas tiene múltiples sitios de accción. Estrobilurinas (Inhibidores Qo, QoI) actúan sobre el sitio de unión quinónico en el complejo del citocromo bcl en la membrana mitocondrial. Triazolpirimidinas no tiene un modo de acción aun conocido (FRAC, 2007). ${ }^{3}$ Concentraciones expresadas como ingrediente activo (a.i.) y fueron suspendidos en agua antes de su uso. 
obtaining $75 \%$ or higher control efficacy. Postinfection activity was only studied after drop application of each fungicide because it was assumed that immersion would have washed off the spores. Therefore, the berries were treated $0,12,24$ and $48 \mathrm{~h}$ after the inoculation with a drop of $12 \mu \mathrm{L}$ of fungicide suspension, which was placed in the inoculation site, at the calyx end of each berry. Berries were then assessed for the diameter of the lesion developed 48-72 $\mathrm{h}$ after the fungicide application.

\section{Design and statistical analyses}

The results for both pre and post infection activity studies were expressed as \% efficacy $\left(\mathrm{E}=100-\left[(100 \mathrm{x}\right.\right.$ treatment $) \cdot$ untreated control $\left.\left.^{-1}\right]\right)$, but $\%$ efficacies were transformed to Probit values prior to analysis.

Considering that $48 \mathrm{~h}$ post-treatment disease incidence was over $50 \%$ for each pathogen, the efficacy of the pre-infection activity obtained after $48 \mathrm{~h}$ post-treatment were studied. For each pathogen, treatments were arranged as completely randomized design with a $2 \times 8$ (application methods $\mathrm{x}$ fungicides) factorial arrangement of treatments. Four replicates and eight berries as an experimental unit were used. Results were subjected to analysis of

Table 2. Effect of fungicide, application method, and the interaction between fungicide and application method on the efficacy of pre-infection fungicide treatments on berries with their pedicels intact of Thompson Seedless table grapes.

Cuadro 2. Efecto del fungicida, del método de aplicación y de la interacción entre fungicidas y métodos de aplicación en la eficacia de los tratamientos fungicidas de pr-einfección, aplicados en bayas con pedicelos de uvas Thompson Seedless.

\begin{tabular}{|c|c|c|c|c|c|}
\hline Source of variation & d.f. & SS & MS & $\mathrm{F}$ & $\mathrm{p}$ \\
\hline & \multicolumn{5}{|c|}{ Aspergillus niger } \\
\hline Method (M) & 1 & 0.863 & 0.863 & 2.3 & 0.136 \\
\hline Fungicide (F) & 7 & 22.665 & 3.238 & 8.633 & $<0.001$ \\
\hline $\mathrm{M} \times \mathrm{F}$ & 7 & 3.498 & 0.5 & 1.332 & 0.256 \\
\hline Residual & 48 & 18.002 & 0.375 & & \\
\hline \multirow[t]{2}{*}{ Total } & 63 & 45.027 & 0.715 & & \\
\hline & \multicolumn{5}{|c|}{ Botrytis cinerea } \\
\hline Method (M) & 1 & 19.25 & 19.25 & 106.898 & $<0.001$ \\
\hline Fungicide $(\mathrm{F})$ & 7 & 9.057 & 1.294 & 7.185 & $<0.001$ \\
\hline$M \times F$ & 7 & 2.118 & 0.303 & 1.68 & 0.137 \\
\hline Residual & 48 & 8.644 & 0.18 & & \\
\hline \multirow[t]{2}{*}{ Total } & 63 & 39.068 & 0.62 & & \\
\hline & \multicolumn{5}{|c|}{ Cladosporium herbarum } \\
\hline Method (M) & 1 & 2.337 & 2.337 & 10.665 & 0.002 \\
\hline Fungicide (F) & 7 & 15.466 & 2.209 & 10.082 & $<0.001$ \\
\hline $\mathrm{M} \times \mathrm{F}$ & 7 & 3.09 & 0.441 & 2.015 & 0.072 \\
\hline Residual & 48 & 10.518 & 0.219 & & \\
\hline \multirow[t]{2}{*}{ Total } & 63 & 31.412 & 0.499 & & \\
\hline & \multicolumn{5}{|c|}{ Penicillium expansum } \\
\hline Method (M) & 1 & 6.119 & 6.119 & 16.954 & $<0.001$ \\
\hline Fungicide $(\mathrm{F})$ & 7 & 58.531 & 8.362 & 23.165 & $<0.001$ \\
\hline$M \times F$ & 7 & 3.922 & 0.56 & 1.552 & 0.173 \\
\hline Residual & 48 & 17.326 & 0.361 & & \\
\hline \multirow[t]{2}{*}{ Total } & 63 & 85.898 & 1.363 & & \\
\hline & \multicolumn{5}{|c|}{ Rhizopus stolonifer } \\
\hline Method (M) & 1 & 9.09 & 9.09 & 30.99 & $<0.001$ \\
\hline Fungicide (F) & 7 & 20.312 & 2.902 & 9.892 & $<0.001$ \\
\hline$M \times F$ & 7 & 17.874 & 2.553 & 8.705 & $<0.001$ \\
\hline Residual & 48 & 14.08 & 0.293 & & \\
\hline Total & 63 & 61.356 & 0.974 & & \\
\hline
\end{tabular}

${ }^{1}$ Treatments were drop $\left(12 \mu \mathrm{L} \cdot\right.$ berry $\left.^{-1}\right)$ applications and immersion for $60 \mathrm{~s}$.

${ }^{\prime}$ Los tratamientos fueron en gotas $\left(12 \mu \mathrm{L} \cdot\right.$ baya $\left.^{-1}\right)$ e inmersión por $60 \mathrm{~s}$. 
variance followed by a multiple comparison test according to Tukey ( $\mathrm{p}<0.05$ ) using Sigmastat (Systat Software, Inc., USA). The relation between time of pre-infection activity and fungicide efficacy were study by a linear regression analysis.

The pre- and post-infection activity of the fungicide treatments were estimated, independently for each pathogen, by a linear regression analysis. Treatments were replicated four times with eight berries as experimental units.

\section{Results}

\section{Pre-infection activity}

The pre-infection activity of the fungicides varied considerably among pathogens. However, it was significantly $(\mathrm{p}<0.001)$ affected by the fungicide used and, with one exception $(A$. niger), it was also significantly affected by the application method (immersion and localized drop applications) $(\mathrm{p}<0.002)$ when evaluation was made $48 \mathrm{~h}$ after treatment. The interaction between fungicide and application method was only significant $(\mathrm{p}<0.001)$ for $R$. stolonifer (Table 2).

In general, pre-infection activity gave 0 to 4 days protection when fungicides were tested as drops placed on the inoculation site, and varied between 0 and 21 days when berries were treated by $60 \mathrm{~s}$ immersion (Table 3 ). The maximum pre-infection activity required to obtain $\geq 75 \%$ control efficacy was estimated by linear regression analysis (data not presented). For example, pre-infection activity of cyprodinil plus fludioxonil protected berries for 4 days against $A$. niger, $B$. cinerea, $P$. expansum and $R$. stolonifer, and only 2 days against $C$. herbarum when this fungicide mixture was applied by drops. Using the immersion treatments, this same fungicide mixture provided 14 days protection against $B$. cinerea, 21 days against $P$. expansum, $R$. stolonifer and A. niger and $<2$ days against $C$. herbarum (Table 3 ).

All the fungicides controlled $B$. cinerea and $C$. herbarum with control efficiencies higher than 78.8 and $82.3 \%$, respectively, when inoculations were made $48 \mathrm{~h}$ after drop applications of fungicides. However, the efficacy of most of these fungicides decreased considerably after immersion treatments. For example, captan had $88.6 \%$ control efficacy against $B$. cinerea after drop applications; this decreased to $45.3 \%$ after immersion treatments (Table 3 ).

Independent from the application method, preinfection applications of boscalid were effective (mean control efficacy 95.3\%) in control of $A$. niger, but were relatively ineffective against $P$. expansum (66.6\% control efficacy) and $R$. stolonifer (63.9\% control efficacy). However, the pre-infection activity of boscalid against $P$. expansum improved significantly when this fungicide was combined with pyraclostrobin or kresoxim methyl (99.1 and 91.9\% control efficacy, respectively).Pre-infectionapplications of BAS $600 \mathrm{KBF}$ in combination with boscalid, metrafenone or pyraclostrobin were weak or ineffective in control of P. expansum (Table 3).

Cyprodinil combined with fludioxonil efficiently controlled $B$. cinerea, A. niger, $P$. expansum and $R$. stolonifer, with mean efficiencies $>89.4 \%$, but it was relatively weak in control of $C$. herbarum $(67.1 \%)$. Preinfection drop applications of captan were effective in control of $B$. cinerea and arrested the development of decay caused by A. niger, $C$. herbarum, and P. expansum. Nevertheless, the same concentration of captan was ineffective against $B$. cinerea after immersion treatments. Regardless of the application method, captan was ineffective against $R$. stolonifer (Table 3 ).

\section{Post-infection activity}

The post-infection activity (curative action) varied among pathogens and fungicide treatments. Nevertheless, this was always short, below $24 \mathrm{~h}$ (Table 3). The maximum curative action (24 h) of cyprodinil in combination with fludioxonil was obtained against $A$. niger and $P$. expansum. However, the post-infection activity for the same treatment was estimated as $0,<12$ $\mathrm{h}$, and $12 \mathrm{~h}$ against $C$. herbarum, $B$. cinerea, and $R$. stolonifer, respectively. Similarly, captan had no curative action against $R$. stolonifer, $<12$ $\mathrm{h}$ against $B$. cinerea and $P$. expansum, and $24 \mathrm{~h}$ against $A$. niger and $C$. herbarum. 
Table 3. Efficacy and pre and post infection activity of new fungicides against Botrytis cinerea, Penicillium expansum, Rhizopus stolonifer, Aspergillus niger and Cladosporium herbarum determined on detached mature berries of Thompson Seedless table grapes.

Cuadro 3. Eficacia y actividad de pre y post infección de nuevos fungicidas contra Botrytis cinerea, Penicillium expansum, Rhizopus stolonifer, Aspergillus niger y Cladosporium herbarum, determinada en bayas cortadas y maduras de uvas Thompson Seedless .

\begin{tabular}{|c|c|c|c|c|c|c|c|}
\hline \multirow[t]{2}{*}{$\begin{array}{l}\text { Fungicide } \\
\text { treatments }\end{array}$} & \multirow[t]{2}{*}{$\begin{array}{l}\text { Concentration } \\
\mathrm{mg} \cdot \mathrm{L}^{-1} \text {, a.i. }\end{array}$} & \multicolumn{3}{|c|}{ Efficacy $^{1}$} & \multicolumn{2}{|c|}{$\begin{array}{c}\text { Estimated } \\
\text { pre-infection activity }\end{array}$} & \multirow{2}{*}{$\begin{array}{c}\begin{array}{c}\text { Estimated } \\
\text { activity }^{3}\end{array} \\
\begin{array}{c}\text { Drop } \\
\text { h }\end{array}\end{array}$} \\
\hline & & $\begin{array}{c}\text { Drop }^{4} \\
\%\end{array}$ & $\begin{array}{c}\text { Immersion }^{4} \\
\%\end{array}$ & $\begin{array}{c}\operatorname{Mean}^{5} \\
\%\end{array}$ & $\begin{array}{l}\text { Drop } \\
\text { days }\end{array}$ & $\begin{array}{l}\text { Immersion } \\
\text { days }\end{array}$ & \\
\hline \multicolumn{8}{|c|}{ Aspergillus niger } \\
\hline $\begin{array}{l}\text { BAS } 600 \mathrm{KBF} \\
+ \text { metrafenone }\end{array}$ & $100+150$ & 63.9 & 64.6 & $64.3 c^{6}$ & 0 & 0 & 0 \\
\hline $\begin{array}{l}\text { BAS } 600 \mathrm{KBF} \\
\text { + boscalid }\end{array}$ & $200+300$ & 99.3 & 91.7 & $95.5 \mathrm{a}$ & 4 & 21 & 24 \\
\hline $\begin{array}{l}\text { BAS } 600 \mathrm{KBF} \\
+ \text { pyraclostrobin }\end{array}$ & $100+100$ & 69.3 & 70.1 & $69.7 b c$ & $<2$ & $<2$ & 24 \\
\hline Boscalid & 600 & 100 & 90.5 & $95.3 \mathrm{a}$ & 4 & 21 & 24 \\
\hline Boscalid & $200+100$ & 964 & 057 & $961 \mathrm{a}$ & 4 & 21 & 24 \\
\hline $\begin{array}{l}\text { + pyraclostrobin } \\
\text { Boscalid }\end{array}$ & $200+100$ & 96.4 & 95.1 & $96.1 \mathrm{a}$ & 4 & 21 & 24 \\
\hline $\begin{array}{l}\text { + kresoxim methyl } \\
\text { Cyprodinil }\end{array}$ & $100+200$ & 87.9 & 97.0 & $92.5 \mathrm{a}$ & 4 & 21 & 24 \\
\hline + fludioxonil & $60+40$ & 99.3 & 97.6 & $98.5 \mathrm{a}$ & 4 & 20 & 24 \\
\hline Captan & 400 & 97.1 & 85.3 & $91.2 \mathrm{ab}$ & 4 & 2 & 24 \\
\hline \multicolumn{8}{|c|}{ Botrytis cinerea } \\
\hline $\begin{array}{l}\text { BAS } 600 \mathrm{KBF} \\
+ \text { metrafenone }\end{array}$ & $100+150$ & 78.85 & 30.95 & $54.9 \mathrm{~d}^{6}$ & 4 & 0 & $<12$ \\
\hline $\begin{array}{l}\text { BAS } 600 \mathrm{KBF} \\
+ \text { boscalid }\end{array}$ & $200+300$ & 90.6 & 72.6 & $81.6 \mathrm{ab}$ & 4 & $<2$ & $<12$ \\
\hline $\begin{array}{l}\text { BAS } 600 \mathrm{KBF} \\
+ \text { pyraclostrobin }\end{array}$ & $100+100$ & 70.3 & 43.3 & $56.8 \mathrm{~cd}$ & 4 & 0 & $<12$ \\
\hline Boscalid & 600 & 77.2 & 57.0 & $67.1 \mathrm{bcd}$ & 2 & $<2$ & $<12$ \\
\hline $\begin{array}{l}\text { Boscalid } \\
+ \text { pyraclostrobin }\end{array}$ & $200+100$ & 94.8 & 54.0 & 74.4abc & $2-3$ & $<2$ & $<12$ \\
\hline Boscalid & & & & & & & \\
\hline $\begin{array}{l}\text { + kresoxim methyl } \\
\text { Cyprodinil }\end{array}$ & $100+200$ & 91.7 & 60.2 & 76.0abcd & $2-3$ & $<2$ & $<12$ \\
\hline + fludioxonil & $60+40$ & 96.2 & 82.5 & $89.4 \mathrm{a}$ & 4 & $10-14$ & $12-24$ \\
\hline Captan & 400 & 88.6 & 45.3 & $67.0 \mathrm{bcd}$ & 3 & 0 & $<12$ \\
\hline \multicolumn{8}{|c|}{ Cladosporium herbarum } \\
\hline $\begin{array}{l}\text { BAS } 600 \mathrm{KBF} \\
+ \text { metrafenone } \\
\text { BAS } 600 \mathrm{KBF}\end{array}$ & $100+150$ & 82.05 & 67.15 & $75.6 \mathrm{~d} 6$ & 4 & $<2$ & $<12$ \\
\hline $\begin{array}{l}+ \text { boscalid } \\
\text { BAS } 600 \mathrm{KBF}\end{array}$ & $200+300$ & 94.6 & 94.4 & $94.5 \mathrm{ab}$ & 4 & 11 & 24 \\
\hline + pyraclostrobin & $100+100$ & 80.3 & 84.6 & $82.5 \mathrm{bcd}$ & 4 & 5 & 24 \\
\hline Boscalid & 600 & 87.8 & 90.4 & $89.1 \mathrm{abc}$ & $3-4$ & 10 & $<12$ \\
\hline $\begin{array}{l}\text { Boscalid } \\
+ \text { pyraclostrobin }\end{array}$ & $200+100$ & 96.9 & 79.0 & $88.0 \mathrm{abc}$ & 4 & 2 & 24 \\
\hline Boscalid & & & & & & & \\
\hline $\begin{array}{l}\text { + kresoxim methyl } \\
\text { Cyprodinil }\end{array}$ & $100+200$ & 88.8 & 68.3 & $78.6 \mathrm{~cd}$ & 4 & $<2$ & $<12$ \\
\hline + fludioxonil & $60+40$ & 82.6 & 51.5 & $67.1 \mathrm{~d}$ & $2-3$ & $<2$ & $<12$ \\
\hline Captan & 400 & 98.9 & 94.0 & $96.5 \mathrm{a}$ & 4 & 12 & 24 \\
\hline
\end{tabular}




\begin{tabular}{|c|c|c|c|c|c|c|c|}
\hline \multicolumn{8}{|c|}{ Penicillium expansum } \\
\hline $\begin{array}{l}\text { BAS } 600 \mathrm{KBF} \\
+ \text { metrafenone }\end{array}$ & $100+150$ & 13.05 & 41.45 & $27.2 c^{6}$ & 0 & 0 & 0 \\
\hline BAS $600 \mathrm{KBF}$ & & & & & & & \\
\hline $\begin{array}{l}+ \text { boscalid } \\
\text { BAS } 600 \mathrm{KBF}\end{array}$ & $200+300$ & 43.7 & 75.2 & $59.5 b$ & 0.5 & 3 & $<12$ \\
\hline + pyraclostrobin & $100+100$ & 55.0 & 86.7 & $70.9 b$ & 0.5 & 4 & $<12$ \\
\hline Boscalid & 600 & 54.1 & 79.0 & $66.6 \mathrm{~b}$ & 0.5 & 3 & $<12$ \\
\hline Boscalid & & & & & & & \\
\hline + pyraclostrobin & $200+100$ & 99.4 & 98.7 & $99.1 \mathrm{a}$ & 4 & 20 & 24 \\
\hline $\begin{array}{l}\text { Boscalid } \\
+ \text { kresoxim methyl }\end{array}$ & $100+200$ & 88.0 & 95.8 & $91.9 \mathrm{ab}$ & 4 & 10 & $<12$ \\
\hline $\begin{array}{l}\text { Cyprodinil } \\
\text { + fludioxonil }\end{array}$ & $60+40$ & 100 & 98.5 & $99.3 \mathrm{a}$ & 4 & 21 & 24 \\
\hline Captan & 400 & 67.8 & 84.8 & $76.3 b$ & nt & 5 & $<12$ \\
\hline \multicolumn{8}{|c|}{ Rhizopus stolonifer } \\
\hline $\begin{array}{l}\text { BAS } 600 \mathrm{KBF} \\
+ \text { metrafenone }\end{array}$ & $100+150$ & $90.6 \mathrm{ab}^{6}$ & $18.6 b^{6}$ & 54.6 & 4 & $<2$ & 0 \\
\hline $\begin{array}{l}\text { BAS } 600 \mathrm{KBF} \\
+ \text { boscalid }\end{array}$ & $200+300$ & $92.2 \mathrm{ab}$ & $26.9 \mathrm{~b}$ & 55.4 & 4 & $<2$ & 0 \\
\hline $\begin{array}{l}\text { BAS } 600 \mathrm{KBF} \\
+ \text { pyraclostrobin }\end{array}$ & $100+100$ & $73.8 \mathrm{bc}$ & $72.5 \mathrm{a}$ & 73.15 & 4 & $<2$ & 0 \\
\hline Boscalid & 600 & $74.7 \mathrm{bc}$ & $53.1 \mathrm{ab}$ & 63.9 & $<1$ & $<2$ & 0 \\
\hline $\begin{array}{l}\text { Boscalid } \\
+ \text { pyraclostrobin }\end{array}$ & $200+100$ & $66.6 \mathrm{bc}$ & $84.6 \mathrm{a}$ & 75.6 & 1 & 6 & 0 \\
\hline Boscalid & & & & & & & \\
\hline $\begin{array}{l}\text { + kresoxim methyl } \\
\text { Cyprodinil }\end{array}$ & $100+200$ & $63.6 \mathrm{bc}$ & $81.9 \mathrm{a}$ & 72.8 & 0 & 3 & 0 \\
\hline + fludioxonil & $60+40$ & $98.5 \mathrm{a}$ & $85.9 \mathrm{a}$ & 92.2 & 4 & 21 & 12 \\
\hline Captan & 400 & $43.8 \mathrm{c}$ & $26.6 \mathrm{~b}$ & 35.2 & 0 & 0 & 0 \\
\hline
\end{tabular}

${ }^{1}$ Efficacy $(\mathrm{E})$ was defined as $(\mathrm{E}=100-[(100 \mathrm{x}$ treatment $) \cdot$ untreated control-1] $)$, determined $48 \mathrm{~h}$ after drop and immersion treatments.

${ }^{2}$ Pre-infection (protectant) activity was defined as time between application and inoculation to obtain $\geq 75 \%$ efficacy. Estimations were made by linear regression analysis. Drop and immersion applications were studied up to 4 and 21 days, respectively.

${ }^{3}$ Post-infection (curative) activity was defined as time between inoculation and fungicide treatments to obtain $\geq 75 \%$ efficacy after drop applications. Estimations were made by linear regression analysis. Drop applications were studied up to 4 days.

${ }^{4}$ Treatments were drop $\left(12 \mu \mathrm{L} \cdot\right.$ berry $\left.^{-1}\right)$ applications and immersion treatment for $60 \mathrm{~s}$.

${ }^{5}$ The interactions between application methods and fungicide treatments were only significant for $R$. stolonifer (Table 2); therefore, the average of the \% efficacy obtained after drop and immersion applications were compared.

${ }^{6}$ Means of four replicates followed by the same letters in each column for each pathogen were not significantly different according to Tukey $(\mathrm{p}=0.05) . \mathrm{nt}=$ not tested.

${ }^{l}$ Eficacia $(E)$ se definió como $E=100-[(100 x$ tratamiento $) \cdot c o n t r o l$ sin tratar-1]), determinado 48 h luego de la aplicación en gotas e inmersión.

${ }^{2}$ Actividad de pre-infección (protectora) se definió como el tiempo entre la aplicación y la inoculación de modo de obtener una eficacia $\geq 75 \%$. Estimaciones realizadas por análisis de regresión lineal. Las aplicaciones en gota e inmersión se estudiaron respectivamente hasta 4 y 21 dias postratamiento.

${ }^{3}$ Actividad de post-infección (curativa) se definió como el tiempo entre la inoculación y la aplicación del fungicida, via gota o por inmersión, de modo de obtener una eficacia $\geq 75 \%$. Estimaciones realizadas por análisis de regresión lineal. Las aplicaciones en gota se estudiaron hasta 4 dias postratamiento.

${ }^{4}$ Tratamientos en gotas (12 $\mu \mathrm{L} \cdot$ baya-1) e inmersión por $60 \mathrm{~s}$.

${ }^{5}$ La interacción entre el método de aplicación y los fungicidas fue solamente significativa en el caso de R. stolonifer (Cuadro 2); por lo tanto, se comparó el porcentaje de eficacia promedio obtenido para tratamientos vía gota e inmersión.

${ }^{6}$ Promedios de cuatro repeticiones seguidos por iguales letras en cada columna no son significativamente diferentes de acuerdo con Tukey $(p=0,05)$. $n t=$ no estudiado.

A $24 \mathrm{~h}$ post-infection activity against $A$. niger, $C$. herbarum, but lack of activity against $B$. cinerea, $P$. expansum and $R$. stolonifer was obtained after drop applications of boscalid (alone or combined with BAS $600 \mathrm{KBF}$, pyraclostrobin or kresoxim methyl) and BAS $600 \mathrm{KBF}$ combined with metrafenone (Table 3).

\section{Discussion}

Production of Chilean table grapes for international markets requires the use of fungicide treatments as one of the main components for disease management. This allows controlling rots caused by $B$. cinerea 
and other filamentous fungi that are commonly present in the vineyards, causing decays before and after harvest (Hewitt, 1988; Pszczolkowski et al., 2001; Latorre, 2002b, 2004; Franck et al., 2005; Donoso and Latorre, 2006).

Several studies have been conducted on the efficacy and activity of various fungicides against other plant pathogenic fungi (O'Leary et al., 1984; Poblete and Latorre, 2001; Wong and Wilcox, 2001; Ferreira et al., 2006; Holb and Schnabel, 2007; Rebollar-Alviter et al., 2007). However, to our knowledge this is the first report regarding pre- and postinfection activity of boscalid and cyprodinil in combination with fludioxonil, BAS $600 \mathrm{KBF}$ and captan against filamentous fungi affecting mature table grapes.

Our study demonstrated important differences in the pre- and post-infection activity of the fungicides evaluated. Coincident with previous reports on other fruits and pathogens, protective applications of these fungicides were more effective in reducing decay incidence than curative applications (Holb and Schnabel, 2007). All the fungicides tested had a null or low $(<24 \mathrm{~h})$ post-infection activity against filamentous fungi causing rots on mature Thompson Seedless table grapes. The high inoculum concentrations used in these studies and the fast development of these rots can possibly explain these results. It has been demonstrated that symptoms of gray mold, caused by B. cinerea, appeared 12- to 24 $\mathrm{h}$ post-inoculation at optimal temperature and humidity (Latorre and Rioja, 2002). Therefore, curative treatments are unlikely to be useful for control decays under field conditions. However, fungicides that do not perform well in our laboratory tests may still do better under field conditions, because in the field, post-infection activity affects a range of pathogen activities including germination of spores deposited on the surface of berry after fungicide applications. Additionally, it is unlikely that grapes would be exposed to the high inoculum concentration as it was always used in these tests.

Similar to previous reports, these results provided additional evidence regarding the high effectiveness of $600 \mathrm{mg} \cdot \mathrm{L}^{-1}$ of boscalid and $60 \mathrm{mg} \cdot \mathrm{L}^{-1}$ of cyprodinil in combination with $40 \mathrm{mg} \cdot \mathrm{L}^{-1}$ fludioxonil against $B$. cinerea on table grapes and other fruit crops (Forster and Staub, 1996; Blacharski et al., 2001; Latorre et al., 2001; Sholberg et al., 2003; Sallato et al., 2007; Wedge et al., 2007). Cyprodinil plus fludioxonil effectively controlled $P$. expansum, $R$. stolonifer and A. niger, but was ineffective in controlling $C$. herbarum. It is interesting that a relatively low concentration of cyprodinil in combination with fludioxonil was used in this study. Therefore, it is possible that using higher concentrations a better control efficacy against these fungi could be obtained.

With one exception, $R$. stolonifer, $100 \mathrm{mg} \cdot \mathrm{L}^{-1}$ of boscalid combined with $200 \mathrm{mg} \cdot \mathrm{L}^{-1}$ of kresoxim methyl, and $200 \mathrm{mg} \cdot \mathrm{L}^{-1}$ boscalid combined with $100 \mathrm{mg} \cdot \mathrm{L}^{-1}$ of pyraclostrobin effectively protected berries against $B$. cinerea and it prevented the development of rot caused by A. niger, C. herbarum, P. expansum and $R$. stolonifer (Forster and Staub, 1996; Latorre et al., 2001; Franck et al., 2005; Rosslenbroich and Stuebler, 2000; Wedge et al., 2007).

Based on this research, the efficacy of the fungicide protectant activity varied with application method when this effect was tested $48 \mathrm{~h}$ post-treatment. In most cases, a better control was obtained with localized drop applications. These differences were mainly attributed to the imperfect coverage of the berry surface obtained by immersion applications. On the contrary, drop applications allowed direct contact of the pathogen with the fungicide deposits. At the same time, the drop applications could possibly facilitate the product absorption by the berry, improving the degree of control obtained.

Therefore, the localized drop application in detached table grape berries was an efficient and reproducible methodology which allowed us to study the in vivo effectiveness of new fungicides. Nevertheless, these results also suggest the need to evaluate these treatments according to the commercial application method before establishing a recommendation. A similar methodology has previously been used to study the effectiveness of these and other fungicides in table grapes and strawberries (Franck et al., 2005; Sallato et al., 2006; Holb and Schnabel, 2007). 


\section{Resumen}

Las enfermedades de pre y postcosecha limitan la producción y exportación de uva de mesa (Vitis vinifera $\mathrm{L}$.) en Chile. Especialmente importante es la pudrición gris (Botrytis cinerea). Además, son frecuentes las pudriciones causadas por Aspergillus niger, Cladosporium herbarum, Penicillium expansum y Rhizopus stolonifer. Este trabajo tuvo el propósito de estudiar, en bayas de uvas Thompson Seedless, la actividad de pre y post-infección de nuevos fungicidas. Con este propósito se empleó bayas maduras ( $<16 \%$ sólidos solubles) con pedicelos intactos. Cada baya se inoculó en el extremo calicinal, depositando $20 \mu \mathrm{L}$ de una suspensión de $10^{6}$ esporas $\cdot \mathrm{mL}^{-1}$ sobre tres heridas practicadas asépticamente con una aguja hipodérmica estéril. Los productos y concentraciones empleadas por litro fueron: boscalid (600 $\mathrm{mg})$, boscalid $(200 \mathrm{mg})+$ pyraclostrobin $(100$ $\mathrm{mg})$, boscalid $(200 \mathrm{mg})+$ kresoxim metil $(100$ $\mathrm{mg})$, cyprodinil $(60 \mathrm{mg})+$ fludioxonil $(40 \mathrm{mg})$, BAS $600 \mathrm{KBF}(100 \mathrm{mg})+$ metrafenone $(150$ $\mathrm{mg})$, BAS $600 \mathrm{KBF}(200 \mathrm{mg})+$ boscalid (300 $\mathrm{mg})$, BAS $600 \mathrm{KBF}(100 \mathrm{mg})+$ pyraclostrobin (100 mg), y captan (400 mg). Cada fungicida se aplicó vía gota $\left(12 \mu \mathrm{L} \cdot\right.$ baya $\left.^{-1}\right)$ depositada sobre tres heridas practicadas en cada baya con una aguja hipodérmica estéril o por inmersión durante $60 \mathrm{~s}$. Luego las bayas se incubaron en cámaras húmedas a $20^{\circ} \mathrm{C}$. La actividad de pre-infección varió considerablemente entre patógeno y dependió significativamente ( $p$ $<0.001$ ) del fungicida usado y con sólo una excepción (A. niger), el método de aplicación tuvo un efecto significativo $(\mathrm{p}<0.002)$. La relación entre fungicida y método de aplicación, determinado a las $48 \mathrm{~h}$ post-tratamiento, fue significativo $(\mathrm{p}<0.001)$ sólo para $R$. stolonifer. En general, la actividad de pre-infección otorgó una protección entre 0 y 4 días al aplicar cada producto en gota y entre 0 y 21 días luego de aplicaciones por inmersión. La actividad de post-infección (acción curativa) varió entre patógenos y dependió del fungicida aplicado. Sin embargo, ésta fue siempre inferior a $24 \mathrm{~h}$.

Palabras clave: Acción curativa, acción preventiva, fungicidas, moho azul, moho gris pudrición ácida,Vitis vinifera.

\section{Acknowledgements}

Authors are very grateful for the financial support received from BASF Chile and Vinnova project Innova 05CTE-01-098.

\section{References}

Adaskaveg, J.E. H. Förster, W.D. Gubler, B. L.Teviotdale, D.F.Thompson. 2005. Reducedrisk fungicides help manage brown rot and other fungal diseases of stone fruit. California Agriculture 59:109-114.

Blacharski, R.W., J.A. Bartz, C.L. Xiao, and D.E. Legard. 2001. Control of postharvest Botrytis fruit rot with preharvest fungicide applications in annual strawberry. Plant Disease 85:597-602.

Donoso, A., and B. A. Latorre. 2006. Characterization of blue mold caused by Penicillium spp. in cold stored table grapes. Cien. Inv. Agr. (on line) 33:119-130.

Errampalli, D., and N. Crnko. 2004. Control of blue mold caused by Penicillium expansum on apples 'Empire' with cyprodinil and fludioxonil. Canadian Journal of Plant Pathology 26:70-75.

Ferreira, E.M., A.C. Alfenas, L.A. Maffia, R.G. Mafia. 2006. Efficiency of systemic fungicides for control of Cylindrocladium candelabrum in eucalypt. Fitopatol. Bras. 31: 468-475.

Forster, B., and T. Staub. 1996. Basis for use strategies of anilinopyrimidine and phenylpyrrole fungicides against Botrytis cinerea. Crop Protection 15:529-537.

Förster, H., G.F. Driever, D.C. Thompson, and J.E. Adaskaveg. 2007. Postharvest decay management for stone fruit crops in California using the "reduced-risk" fungicides fludioxonil and fenhexamid. Plant Disease 91:209-215.

FRAC. 2007. FRAC code list 2: Fungicides sorted by modes of action. Fungicide Resistance Action Committee. www.frac.info/frac/index. htm. Accessed, April 27, 2007.

Franck, J., B. A. Latorre, R. Torres, and J. P. Zoffoli. 2005. The effect of preharvest fungicide and postharvest sulfur dioxide use on postharvest decay of table grapes caused by Penicillium expansum. Postharvest Biology and Technology 37:20-30.

Hewitt, W.B. 1988. Berry rots and raisin molds. p. 26-28. In: R.C. Pearson and A.C. Goheen (eds.). Compendium of Grape Diseases. The American Phytopathological Society. St. Paul MN, USA.

Holb, I.J., and G. Schnabel. 2007. Differential effect of triazoles on mycelial growth and disease measurements of Monilinia fructicola isolates with reduced sensitivity to DMI fungicides. Crop Protection 26:753-759. 
Jones, A. L. y Latorre, B. A. 1985. Algunas consideraciones sobre el uso de los fungicidas modernos. Revista Frutícola (Chile) 6:21-23.

Latorre, B.A. 2004. Enfermedades de las Plantas Cultivadas. Sexta ed. Ediciones Universidad Católica, Santiago, Chile. 638 pp.

Latorre, B. A., V. Flores, A. M. Sara and A. Roco. 1994. Dicarboximide resistant, isolates of Botrytis cinerea from table grapes in Chile: survey and characterization. Plant Disease 78: 990-994.

Latorre, B. A., C. Lillo, y M. E. Rioja. 2001. Eficacia de los tratamientos fungicidas para el control de Botrytis cinerea de la vid en función de la época de aplicación. Cien. Inv. Agr. 28:61-66.

Latorre, B. A. y M. Rioja. 2002. El efecto de la temperatura y de la humedad relativa sobre la germinación de conidias de Botrytis cinerea. Cien. Inv. Agr. 29:67-71.

Latorre, B. A., I. Spadaro, and M. E. Rioja. 2002a. Occurrence of resistant strains of Botrytis cinerea to anilinopyrimidine fungicides in table grapes in Chile. Crop Protection 21:957-961.

Latorre, B. A., S. C. Viertel, and I. Spadaro. 2002b. Severe outbreaks of bunch rots caused by Rhizopus stolonifer and Aspergillus niger on table grape in Chile. Plant Disease 86:815.

Lydakis, D., and J. Aked. 2003. Vapour heat treatment of Sultanina table grapes. I: control of Botrytis cinerea. Postharvest Biology and Technology 27: 109-116.

Lichter, A., Y. Zutky, L. Sonego, O. Dvir, T. Kaplunov, P. Varig, and R. Ben-Arie. 2002. Etanol controls postharvest decay of table grapes. Postharvest Biology and Technology 24:301-308.

McGrath, M.T. 2004. What are Fungicides. The Plant Health Instructor. DOI: 10.1094/PHI-I2004-0825-01.

O'Leary, A.L., A.L. Jones, and G.R. Ehret. 1984. Greenhouse evaluation of the curative and protective action of sterol-inhibiting fungicides against apple scab. Phytopathology 74:249252.

Poblete, J.A., and B.A. Latorre. 2001. Pre-infection and curative activity of sterol inhibitor fungicides against Venturia inaequalis on apples. Cien. Inv. Agr. 28:145-150.
Pszczolkowski, Ph., B.A. Latorre y C. Ceppi di Lecco. 2001. Efectos de los mohos presentes en uvas cosechadas tardíamente sobre la calidad de los mostos y vinos Cabernet Sauvignon. Cien. Inv. Agr. 28:157-163.

Rebollar-Alviter, A., L.V. Madden, and M.A. Ellis. 2007. Pre- and post-infection activity of azoxystrobin, pyraclostrobin, mefenoxam, and phosphite against rot of strawberry, caused by Phytophthora cactorum. Plant Disease 91:559564.

Rosslenbroich, H. J and D. Stuebler. 2000. Botrytis cinerea-history of chemical control and novel fungicides for its management. Crop Protection 19:557-561.

Sallato, B. V., and B. A. Latorre. 2006. First report of practical resistance to QoI fungicides in Venturia inaequalis (Apple Scab) in Chile. Plant Disease 90: 375.

Sallato, B.V., R. Torres, J.P. Zoffoli, and B.A. Latorre. 2007. Effect of boscalid on postharvest decay of strawberry caused by Botrytis cinerea and Rhizopus stolonifer. Spanish Journal of Agricultural Research 5:67-78.

Sholberg, P. L., K. E. Bedford and S. Stokes 2003. Effect of preharvest application of cyprodinil on postharvest decay of apples caused by Botrytis cinerea. Plant Disease 87:1067-1071.

Szkolnik, M. 1978. Techniques involved in greenhouse evaluation of deciduous tree fruit fungicides. Ann. Rev. Phytopathol. 16:103-129.

Wedge, D.E., B.J. Smith, J.P. Quebedeaux, and R.J.Constantin. 2007. Fungicide management strategies for control of strawberry fruit rot diseases in Louisiana and Mississippi. Crop Protection 26:1449-1458.

Wong, P.F., and F.W. Wilcox. 2001. Comparative physical modes of action of azoxystrobin, mancozeb, and metalaxyl against Plasmopara viticola (grapevine downy mildew). Plant Disease 85:649-656.

Zahavi, T., L. Cohen, B. Weiss, L. Schena, A. Daus, T. Kaplunov, J. Zutkhi, R. Ben-Arie, and S. Droby. 2000. Biological control of Botrytis, Aspergillus and Rhizopus rots on table and wine grapes in Israel. Postharvest Biology and Technology 20:115-124. 